\title{
Semantic Relevance and Semantic Disorders
}

\author{
Giuseppe Sartori $^{1}$ and Luigi Lombardi $^{2}$
}

\begin{abstract}
Semantic features are of different importance in concept representation. The concept elephant may be more easily identified from the feature $<$ trunk $>$ than from the feature $<$ four legs $>$. We propose a new model of semantic memory to measure the relevance of semantic features for a concept and use this model to investigate the controversial issue of category specificity. Category-specific patients have an impairment in one domain of knowledge (e.g., living), whereas the other
\end{abstract}

\section{INTRODUCTION}

Semantic memory includes knowledge about concepts of various types, such as perceptual information ( $d o g$ : $<$ has four legs $>$ ), functional information $(d o g$ : $<$ is used for hunting $>$ ), associative information (dog: <likes to chase cats $>$ ), and encyclopedic information ( $d o g$ : < may be one of many breeds $>$ ).

Neuropsychological studies conducted on patients with specific knowledge impairments have been a useful source of data for addressing issues about the organization of conceptual-semantic knowledge in the human brain. Semantic memory may be disrupted in a number of ways. In particular, semantic memory impairment may be confined to concepts belonging to certain categories; the most frequent category-specific deficit is that for animals and foodstuffs together with preservation of knowledge about manmade artifacts (Capitani, Laiacona, Mahon, \& Caramazza, 2003). Etiology is quite heterogeneous and includes herpes encephalitis, brain abscess, anoxia, stroke, head injury, and dementia of Alzheimer type (DAT), while lesions typically include the temporal lobes and, in particular, their inferior parts (Capitani et al., 2003).

Many theories have been proposed to explain the corpus of data collected. According to the domainspecific theory, category specificity arises from categorical organization of knowledge in the brain (Caramazza \& Shelton, 1998). Other theories explain category specificity as a property emerging from differential distribution, across categories, of sensory and functional features (Warrington \& Shallice, 1984) or of features that are

\footnotetext{
${ }^{1}$ Università di Padova, ${ }^{2}$ Università di Trento
}

domain (e.g., nonliving) is relatively spared. We show that categories differ in the level of relevance and that, when concepts belonging to living and nonliving categories are equated to this parameter, the category-specific disorder disappears. Our findings suggest that category specificity, as well as other semantic-related effects, may be explained by a semantic memory model in which concepts are represented by semantic features with associated relevance values.

shared among many concepts (Devlin, Gonnerman, Andersen, \& Seidenberg, 1998).

Despite the vast amount of neuropsychological data published over the last two decades, the origin of category-specific deficits remains unclear. Here we show that semantic relevance may be a variable that affects category specificity. In our model, concepts are represented by a vector of semantic features, and relevance is a measure of the contribution of semantic features to the "core" meaning of a concept. The "core" meaning of a concept is thought to include those semantic features that enable to identify the concept (and to discriminate it from other similar concepts). We assume that subjects' verbal description may be used to derive these important features. For example, $<$ has a trunk $>$ is a semantic feature of high relevance for the concept elephant because most subjects use it to define elephant, whereas very few use the same feature to define other concepts. < Has 4 legs $>$, on the other hand, is a semantic feature with lower relevance for the same concept because few subjects use it in the definition of elephant while using it in defining many other concepts.

When a set of semantic features is presented, the overall relevance results from the sum of the individual relevance values associated with each of the semantic features. The concept with the highest summed relevance is the one that will be retrieved. For example, the three features <similar to a goose $>,<$ lives in ponds $>$, and $<$ has a beak $>$ have a total relevance of 4.94 for duck, 3.67 for swan, and 0.74 for ostrich. The retrieved concept will be duck, because it has the highest relevance. It may happen that, in the presence of degraded features of duck, swan is erroneously retrieved as eventually resulting in higher relevance than duck. Stated more plainly, overall accuracy in concept retrieval 
is poor when (a) concepts have low relevance and (b) they have many other semantically similar concepts with which they may be confused.

Relevance-based approaches lie implicitly at the base of a great deal of theorizing about semantic knowledge (Warrington \& Shallice, 1984). Although the idea seems reasonable enough, it has not been instantiated in any explicit model that specifies exactly how relevance is calculated. Available quantitative models of semantic mapping do not assume graded relevance, but rather the presence or absence of a feature (Tversky, 1977). We propose a new model that integrates the standard feature-based semantic memory model with the notion of relevance of a semantic feature (see Methods section) and show that this framework has promising applications in the study of normal and damaged semantics. To anticipate our results, we find that relevance captures the importance of a semantic feature for the meaning of a concept, so that features that are higher in relevance permit the identification of the target concept with higher accuracy.

Our view is that relevance of semantic features may influence the organization of semantic memory. After comparing the predictions of the model to previously published neuropsychological data, we suggest that our model accounts for many of the known facts in semantic memory disorders and, in particular, in categoryspecific deficits. In summary, our results show how relevance of semantic features may be an organizing principle in semantic memory.

\section{RESULTS}

We mapped the relevance of semantic features for 254 concepts ( 43 animals, 15 musical instruments, 53 vegetables, 143 objects) following the procedure described in the Methods section. A vector of semantic features, with corresponding relevance weights, was derived for each of these concepts. Representative results for concepts gull and earrings are reported in Table 1.

Identification of the target concept is easier if semantic features with higher relevance values are presented, and if relevance is important to correct retrieval of a concept, reported dissociations may be predicted by this parameter. To examine whether category differences are due to differences in relevance, the distributional statistics of semantic features and their weights across categories were investigated.

\section{Semantic Features and Categories}

On the database of 254 concepts, we investigated relevance values across categories and across different feature types and found that categories differ in average relevance, as reported in Table 2 and Figure 1.

Results show that living items have lower average relevance with respect to nonliving ones. When relevance is not controlled for, the identification of living is expected to be more difficult than nonliving. This is because the relevance of living most of the time is lower than that of nonliving. In fact, when all concepts are ordered for relevance, the first quartile includes $35 \%$ of nonliving and only $7 \%$ of living items. Instead, the fourth quartile includes $13 \%$ of nonliving and $51 \%$ of living concepts. However, we can also predict the reverse dissociation if nonliving items with lower relevance than living are selected (Sacchett \& Humphreys, 1992).

\section{Perceptual and Functional Semantic Features}

Each semantic feature may be classified as perceptual, functional, or associative-encyclopedic (Caramazza \&

Table 1. Representative Semantic Features of High and Medium Relevance Values for Concepts Gull and Earrings

Features with maximum relevance

$\begin{array}{ll}<\text { found near the sea }> & 1.58 \\ <\text { has webbed feet }> & 1.43 \\ <\text { has wings }> & 0.93\end{array}$

Features with medium relevance

$<$ has white feathers $>$

0.93

$<$ hunts for fish $>$

0.70

$<$ flies $>$

0.67

0.68

.005
Average relevance (all features)

Cosine similarity: $r_{\mathrm{cos}}$ (gull and earrings)

$\begin{array}{ll}1.58 & <\text { found in jewelers' shops }> \\ 1.43 & <\text { may be made of gold }> \\ 0.93 & <\text { may have clips }>\end{array}$

2.32

2.21

2.00

All analyses reported hereafter are based on relevance values computed on the full set of 254 concepts. The cosine measure of similarity $\left(r_{\text {cos }}\right)$ between the two features vector for concepts gull and earrings is also reported. The cosine is a popular measure for similarity between text vectors (see Kintsch, 2001). An important property is that it does not depend from the number of semantic features for different concepts. 
Table 2. Descriptive Statistics of Average Relevance Values of Concepts Belonging to Differing Categories

\begin{tabular}{lcrr}
\hline Category & Mean Relevance & Standard Deviation & Range \\
\hline Living $(n=96)$ & 0.75 & 0.005 & $0.64-0.91$ \\
Vegetables $(n=53)$ & 0.73 & 0.004 & $0.64-0.85$ \\
Animals $(n=43)$ & 0.77 & 0.005 & $0.64-0.91$ \\
Nonliving $(n=158)$ & 0.82 & 0.007 & $0.68-1.09$ \\
Objects $(n=143)$ & 0.82 & 0.007 & $0.68-1.09$ \\
Musical instruments $(n=15)$ & 0.82 & 0.004 & $0.73-0.89$ \\
\hline
\end{tabular}

For every concept, average relevance, using all semantic features appearing in the definition of a concept, was computed. The average of average relevance values for concepts are listed. Bonferroni post hoc comparisons showed that (a) average relevance of living items is lower than that of nonliving items $(p<.001)$, (b) vegetables and animals have lower relevance than objects $(p<.001)$, (c) animals, but not vegetables, have lower relevance than musical instruments $(p<.001)$, and $(d)$ the difference between animals and vegetables fell short of statistical significance. We also calculated relevance of semantic feature of a subset of 25 living and 25 nonliving items matched, across categories, for word frequency (living $=$ 1.72 , nonliving $=1.784 ; p=.1$ ), familiarity (living $=5.22$, nonliving $=5.16 ; p=.39$ ), typicality (living $=4.55$, nonliving $=4.43 ; p=.13$ ), and age of acquisition (living $=3.51$, nonliving $=3.44 ; p=.49$ ). The relevance values of these 50 items, computed when embedded in the full set of 254 items, yielded a lower value for living. Similar results were observed when relevance values of these 50 items was computed using these same 50 items only. In this case, average relevance of living was 0.728 , while that of nonliving was higher with a value of $0.781(p<.003)$. Therefore, computing relevance from a set of living items of the same number as nonliving does not seem to change the basic conclusion that living have on the average lower relevance than nonliving. To ensure that results were not biased by the unequal number of living and nonliving concepts in our set of 254 items, we also analyzed the feature norms published by Garrard, Lambon-Ralph, Hodges, and Patterson (2001) for 30 living and 32 nonliving. Also, in this case, average relevance for living was confirmed to be lower than that for nonliving $(p<.005)$. In the end, semantic relevance seems to capture aspects of concepts that are not measured by other psycholinguistic parameters. The correlation of semantic relevance with frequency is .11 , with familiarity .16, with typicality .11, and with age of acquisition .06.

Shelton, 1998; Warrington \& Shallice, 1984). Perceptual features include ones that may be perceived, such as parts of objects or perceptual properties (e.g., <is sharp $>$ ). Functional features include verbs referring to functions (e.g., <used for lighting up >). Associativeencyclopedic features include examples like < eager for honey $>,<$ typical of summer $>$. The relevance values for these feature types are listed in Table 3.

The living category has more perceptual attributes and fewer functional attributes per concept than nonliving. The relevance values of perceptual and functional

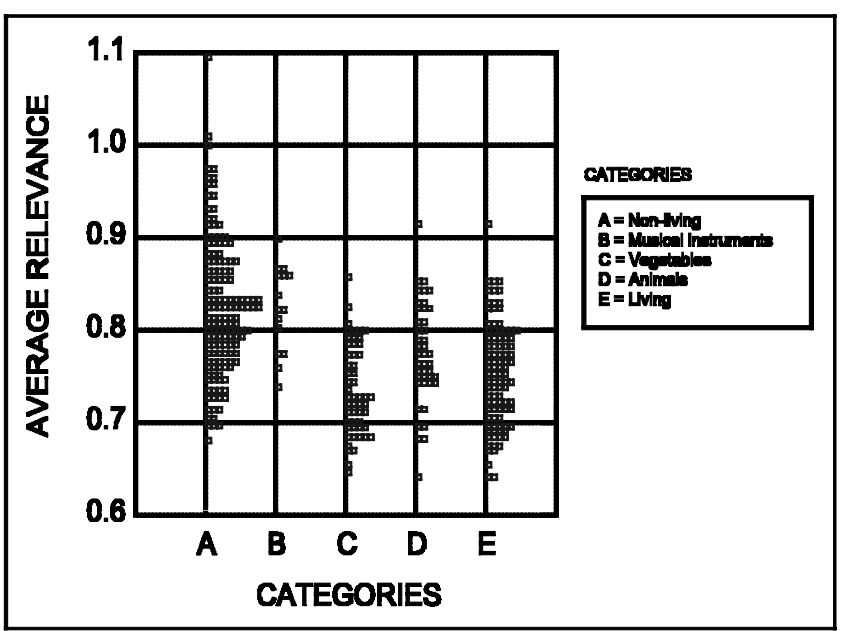

Figure 1. Concepts of different categories with corresponding average relevance. More than half nonliving items have an average relevance above 0.8 ; very few living items are above this value. Musical instruments are reported separately from nonliving. attributes are lower than the corresponding figures for nonliving, particularly for functional attributes. Relevance for perceptual and functional features of living does not differ.

In contrast, nonliving have a higher number of functional attributes and a lower number of perceptual attributes with respect to living. The importance of functional attributes (as measured by relevance) is higher than that of perceptual features. Living and nonliving do not differ with respect to associative-encyclopedic semantic features.

\section{Within-category Similarity}

It has been suggested that high similarity between exemplars of the same category may reduce performance for living and musical instruments (Humphreys \& Forde, 2001).

We analyzed similarities between pairs of concepts by calculating the standardized cosine $\left(r_{\text {cos }}\right)$ between vectors of semantic attributes. We applied this procedure to all pairs of concepts of each category, and an average cosine $\left(\bar{r}_{\text {cos }}\right)$, representing the average similarity between all pairs of exemplars of a given category, was calculated.

Maximum within-category similarity is found among exemplars of musical instruments $\left(\bar{r}_{\mathrm{cos}}=.21\right)$, followed by vegetables $\left.\bar{r}_{\mathrm{cos}}=.11\right)$ and animals $\left(\bar{r}_{\mathrm{cos}}=\right.$ .096). Minimum similarity occurs between pairs of objects $\left(\bar{r}_{\mathrm{cos}}=.033\right)$. Musical instruments have higher within-category similarity than vegetables and animals, $t(66)=14.3, p<.001 ; t(56)=18.28, p<.001$, and 
Table 3. Average Number and Average Relevance of Semantic Attributes (Perceptual, Functional, Associative-Encyclopedic) for Living and Nonliving Items

\begin{tabular}{llll}
\hline Category & \multicolumn{1}{c}{ Perceptual } & Functional & Associative-Encyclopedic \\
\hline Living (a) $(n=96)$ & Number $=26.4$ & Number $=7.3$ & Number $=25.6$ \\
& Relevance $=0.66$ & Relevance $=0.63$ & Relevance $=0.88$ \\
& Standard deviation $=0.06$ & Standard deviation $=0.10$ & Standard deviation $=0.09$ \\
& Number $=16.9$ & Number $=10.1$ & Number $=26.6$ \\
& Relevance $=0.71$ & Relevance $=0.83$ & Relevance $=0.88$ \\
& Standard deviation $=0.11$ & Standard deviation $=0.11$ & Standard deviation $=0.10$
\end{tabular}

t tests: (a) vs. (b), $d f=237$

Number

$11.7, p<.001$

7.30, $p<.001$

$1.11, p=.265$

Relevance

$3.19, p<.001$

13.19, $p<.001$

$0.64, p=.52$

Musical instruments are excluded from nonliving.

the same is true for vegetables and animals when compared with objects, $t(194)=34.87, p<.001$; $t(184)=37.32, p<.001$. Musical instruments therefore are more confusable than other objects, and this may explain why some patients who are spared on objects have impairment on musical instruments (Dixon, Piskopos, \& Schweizer, 2001).

We may summarize the qualitative differences between living, and nonliving in the distribution of semantic features as follows: (a) living have more semantic features with lower relevance than nonliving; (b) living have perceptual and functional semantic features with low relevance; (c) living have high semantic similarity between exemplars; (d) animals and vegetables show similar profiles in terms of relevance and within-category similarity; (e) musical instruments have the same relevance as other objects but very high similarity among exemplars of the category.

\section{Category Specificity and Relevance}

\section{Michelangelo}

If we are correct in assuming that low relevance accounts for inaccuracy in concept retrieval, then we may expect that a category-specific patient, when presented with a set of living and nonliving items matched for relevance for identification, should no longer be selectively impaired.

Michelangelo was the patient selected for this experiment. He underwent extensive investigations and was ideal for our purpose because he has a permanent, severe category-specific deficit for living things that developed after herpes simplex virus encephalitis in 1984, which resulted in bilateral lesions to the medial and inferior parts of the temporal lobes. His semantic deficit did not change over time or after attempts at rehabilitation (Capitani et al., 2003; Sartori, Job, \& Zago, 2002).

We presented the patient with a "naming to verbal description" task. This task was selected because it has the advantage of being directly interpretable within the framework that we propose here. In fact the model, as presented here, is not suited to make predictions on task that require visual processing (e.g., picture naming). In the "naming to verbal description" task, the subject is required to identify the concept corresponding to a spoken description consisting of a set of semantic features. Experimental stimuli were constructed using three semantic features for each target concept. For example, the three features referring to camel were the following: $<$ has two humps $>$, relevance $=3.58$; $<$ lives in the desert $>$, relevance $=1.85$; and $<$ used by the three magi $>$, relevance $=1.58$; total relevance $=7.03$. The nonliving item matched to camel for relevance was bicycle, which was defined by the following three features: $<$ has handlebars $>$, relevance $=2.52$; $<$ two wheels $>$, relevance $=2.27 ;<$ is pedaled $>$, relevance $=2.12$; total relevance $=6.95$. Sixty descriptions, 30 of living and 30 of nonliving items, were derived, all matched for the sum of the relevance values of the three semantic attributes of the description (average sum living $=5.64$, average sum nonliving $=5.65 ; p=.98$ ) According to the model, the absence of any differences in relevance among sentences describing living and nonliving items should be equal to naming accuracy in the two categories. Furthermore, the 30 living and 30 nonliving items were matched for frequency, familiarity, typicality, age of acquisition, name agreement, and concept agreement (minimum probability at $t$ tests comparing all 60 items on these variables, $p=.24$ ). This matching was also important to guarantee that possible differences in performance could not be explained by a bias in one of 
these parameters, which are known to have an effect on recognition (Funnell \& Sheridan, 1992; Stewart, Parkin, \& Hunkin, 1992). Michelangelo was given this naming test with the following results: living $=12 / 30$ (corresponding to a $z=-3.45$, computed using data collected on a group of 6 men aged 65-70), nonliving $=14 / 30$ correct $(z=$ $-3.15)$. Differences between living and nonliving were not significant and fell within the control range.

A second "naming to verbal description task" consisted of 20 descriptions of living and 20 of nonliving concepts. As in the previous test, the relevance of the three semantic features was equated across categories (living $=3.78$, nonliving $=3.74 ; p=.95$ ). Average concept relevance, computed using all the semantic features, was also matched across categories.

The average relevance of all semantic features of living was 0.79 , and did not differ from that of nonliving (.79). Michelangelo produced the correct response to $6 / 20$ $(z=-1.82)$ of living descriptions and $8 / 20(z=$ $-2.94)$ of nonliving. Again, the differences between living and nonliving were not significant and fell within the control range. The results of the two tests show that no differences between the two categories can be detected when relevance is matched across categories.

Last, we analyzed the naming errors produced by Michelangelo in the two tasks, to verify whether errors are facilitated by semantic similarity. Michelangelo made a total of 60/100 naming errors. Of these, 28/60 were wrong responses, which were included in the set of 254 concepts (e.g., duck $\hookrightarrow$ swan) and which could be analyzed for their similarity to the target. Standardized cosine similarity between target and error was significantly higher than the similarity of the same target with the category (which is represented by the similarity of the target with all the other concepts of the category), $F(1,48)=34.27, p<.001$.

These data show that when Michelangelo was given a naming task with semantic features matched for their relevance across categories, his apparent severe impairment for living disappeared. When he produced an error in retrieving a concept after having been given its semantic features, the error consisted of a response that had high semantic similarity with the target.

However, the demonstration that category specificity disappears in Michelangelo relies upon acceptance of the null hypothesis and may be considered inadequate. In the following section, we show that category specificity may be reversed simply by manipulating the relevance values of semantic features.

\section{Dementia of Alzheimer Type}

Six patients with a diagnosis of probable Alzheimer were selected. They showed a category-specific deficit for living, which was significant and consistent across all the four background semantic memory tests used for assessment as reported in the Methods section (see
Table 4). Based on these results, we believe that our six DAT patients may be considered cases of category specificity for living.

We wanted to test our model on these patients by trying to reverse the category specificity for living, and thus developed a third "naming-to-definition" test, organized as follows:

1. Thirty concepts were used (15 living and 15 nonliving); the average relevance for living was 4.42 and for nonliving 4.42 .

2. For each concept, there were high- and lowrelevance descriptions, both with three semantic features. Summed relevance for living and nonliving was matched (living high relevance $=6.55$; living low relevance $=2.29$; nonliving high relevance $=6.55$, nonliving low relevance $=2.30$ ), and high relevance descriptions differed significantly from low ones $(p<.001)$.

3. Each concept was presented twice, half the times, definitions with high relevance were presented first, and in the other, descriptions with low relevance were presented first.

4. Living and nonliving were matched for all other relevant psycholinguistic factors that may impair performance, such as frequency $(p=.79)$, familiarity $(p=$ $.11)$, typicality $(p=.65)$, age of acquisition $(p=.29)$, and name agreement $(p=.97)$.

This test was given to all six category-specific patients and to the control group of 16 subjects, of comparable age and level of education. An ANOVA with group (controls vs. DAT), category (living vs. nonliving), and relevance (high vs. low) showed that normal controls were more accurate than DAT patients, $F(1,20)=89.47$, $p<.001$. The main effect of category was not significant, whereas responses to high relevance items were more accurate than low relevance ones, $F(1,20)=161.33$, $p<.0001$. The relevance effect, high relevance minus low relevance, was greater for patients than for controls, $F(1,20)=13.6, p<.001$, and the same effect was larger for living than for nonliving, $F(1,20)=19.8, p<.001$.

All controls were more accurate when naming high relevance items relative to low ones, and this pattern was observed for both living and nonliving. DAT patients were similar to controls in consistently showing higher accuracy in naming high relevance items, irrespective of category. Only one subject named nonliving with high relevance at the same level of accuracy as low relevance items, but behaved like the others with living.

When we compare performance on living low relevance and nonliving high relevance, patients showed the same advantage for nonliving as that observed in background category-specific tests. Instead, the pattern was completely reversed when considering living high relevance and nonliving low relevance, $t(5)=7, p<.001$. All six patients were more accurate in naming living high relevance items than nonliving low relevance ones. This demonstration complements that for Michelangelo, but 
Table 4. Accuracy of Controls and Six DAT Category-specific Patients on a Naming-to-Definition Task

\begin{tabular}{lccc}
\hline Controls $(n=16)$ & H Relevance $(n=30)$ & L Relevance $(n=30)$ & Category \\
\hline Living $(n=15)$ & $12.69 / 15$ & $9.2 / 15$ & $21.9 / 30$ \\
Nonliving $(n=15)$ & $11.31 / 15$ & $9.65 / 15$ & $20.9 / 30$ \\
Relevance & $24 / 30$ & $18.9 / 30$
\end{tabular}

DAT patients $(n=6)$

Living $(n=15)$

Nonliving $(n=15)$

Relevance
$8.33 / 15$

$6.66 / 15$

$14.99 / 30$
$2.0 / 15$

$10.33 / 30$

3.66/15

$5.66 / 30$

Individual Results

H Relevance

L Relevance

Category Effect

MR

Living
Nonliving

9

6

FR

$$
\text { Living }
$$

Nonliving

MS

$$
\begin{aligned}
& \text { Living } \\
& \text { Nonliving }
\end{aligned}
$$

AE

$$
\text { Living }
$$

Nonliving

RN

$$
\text { Living }
$$

Nonliving

VP

$\begin{array}{llll}\text { Living } & 6 & 0 & \chi^{2}(1) \approx 0, n s \quad 2 \\ \text { Nonliving } & 4 & 2\end{array}$

Controlled factors were category (living, nonliving) and relevance $(\mathrm{H}=$ high, $\mathrm{L}=\mathrm{low})$. Each of the DAT patients did not show the category effect. in this case, it was not based on the acceptance of the null hypothesis and further confirms our conclusion that category specificity may emerge as a side effect of uncontrolled relevance.

\section{DISCUSSION}

Research on category-specific semantic memory disorder has yielded an enormous amount of experimental and clinical data. Current theories about the origin of category-specific semantic impairment are unable to account for all the clinical data. Most theories explain selective semantic impairment as a secondary characteristic determined by the intrinsic differences, in semantic features, of exemplars belonging to different categories.

The sensory functional theory assumes that dissociations between living and nonliving arise from the differential contribution of sensory and functional semantic features among concepts within the two domains. While nonliving items are encoded by both perceptual and functional features, living have very few functional features and are mostly defined by perceptual features (Warrington \& Shallice, 1984). Selective impairment is expected to emerge for living when perceptual features 
are disrupted by brain damage, as nonliving may always benefit from unaffected functional features (Caramazza \& Shelton, 1998; Warrington \& Shallice, 1984). Unlike the sensory/functional theory, our model does not postulate that features are organized according to type, but rather that feature type, as well as category effects, emerge from differences in the relevance of features.

The "correlated features" theory (Durrant-Peatfield, Tyler, Moss, \& Levy, 1997) is based on the assumption that nonliving are characterized by pairs of perceptual and functional features (e.g., <has a blade $>,<$ is used for cutting $>$ ), which reinforce each other in semantic processing. Instead, living items do not have this sort of pairings and, in cases of brain damage, this will cause an advantage for nonliving (Tyler \& Moss, 1997; De Renzi \& Lucchelli, 1994).

The "crowding" theory states that category specificity is a consequence of the higher similarity of living with respect to nonliving. Living may therefore be confused more with other living, rather than nonliving with other nonliving (Sacchett \& Humphreys, 1992).

All these theories focus on explaining the impairment for living, which is the most frequently observed, but they seem to struggle on accounting for the whole corpus of the collected data. In particular, a critical point is the impairment for artifacts that have repeatedly been documented (Capitani et al., 2003; Sacchett \& Humphreys, 1992).

The domain-specific hypothesis assumes that categories are not emerging characteristics but rather determined by brain organization, presumably on an evolutionary basis (Caramazza \& Shelton, 1998). It postulates physically separate and functionally independent stores in the brain for dissociable categories of knowledge. It may easily account for double dissociations but fails to explain, without further assumptions, why general semantic features are better preserved than specific ones (Hodges, Graham, \& Patterson, 1995), or why musical instruments may be impaired as living (Dixon et al., 2001), and in general patterns of breakdowns within domains.

Here, we have taken an alternative approach. In the model we propose, concepts are assumed to be represented within a noncategorical conceptual system, and category-specific deficits emerge as a result of differences in the relevance of concepts, rather than as divisions in independent stores. It assumes, as do other models (Humphreys \& Forde, 2001; Durrant-Peatfield et al., 1997), that concepts are described by a set of semantic features but that each of the constituent semantic features also has an associated relevance weight that is believed to reflect the level of contribution to the "core" meaning of the concept. The model predicts that errors may arise either because of low relevance of semantic features or high similarity with competing concepts. Therefore, at constant values of relevance, concepts that have many other closely similar concepts will be more error-prone.
Relevance is different from distinctiveness as proposed by the conceptual structure theory (e.g., Tyler et al., 2000). Distinctiveness is a dimension that is not concept-dependent since scores are high when the feature is found in only a few concepts. Instead, the relevance of a given semantic feature varies across different concepts, and in a way, may be considered concept-dependent. For example, the feature $<$ has a beak $>$ has a value of 1.21 for the concept duck and of only 0.47 for the concept swan. In this view, our model resembles that suggested by those researchers who advocate that psychologically plausible semantic models should characterize features in a context-sensitive manner. Tversky (1977), for example, proposed a featurebased model of similarity, in which common features tend to increase the perceived similarity of two concepts and feature differences tend to diminish perceived similarity. This author claims that feature salience plays an important role and that it is not fixed, but varies with context. However, his definition of salience is more similar to distinctiveness as described above, than to relevance as proposed by us (e.g., Tversky, 1977, p. 342).

We may assume that damage to the semantic system affects features by reducing the degree to which they are activated. When features with high relevance become unavailable, errors occur in naming these items. The more relevant a particular feature is to a given concept, the more probable that that concept will be misnamed when the feature is damaged and, therefore, the behavioral consequence of damage is proportional to the relevance of the lost feature. Given two prototypical concepts, one living and one nonliving with the same number of features, random damage of the same extent to the two concepts will leave the living concept with a lower residual relevance. If this model is correct, then the damaged knowledge will reproduce the pattern that is observed in the original sample. In other words, if concepts are randomly sampled from categories, then lower performance for living is expected, because living have an average lower relevance. If, on the contrary, relevance is matched across categories, then no difference is expected, and this is exactly what we observed. However, we are not committed to any specific hypothesis about how brain damage may be mimicked by a neural network. Alternative hypotheses on this issue are possible. For example, if we consider a feed-forward neural network (e.g., Small, Hart, Nguyen, \& Gordon, 1995), then features of similar relevance may be captured by the same hidden units. This suggests that focal damage affects specific hidden units and has disproportionate effects on individual categories.

We tested predictions based on our model in two separate investigations, one conducted on a herpes patient and one on six DAT patients. In Michelangelo, the herpes patient, after matching living and nonliving items for relevance, the previously repeatedly documented advantage for nonliving failed to materialize. 
The importance of relevance in the origin of category specificity was further confirmed in the second study, conducted on six category-specific DAT patients, in whom the original category-specific impairment was reversed by manipulating the level of relevance of living and nonliving concepts. In all the six patients who showed impairment for living on preliminary testing, we reported the absence of this impairment and selective impairment for nonliving when living concepts were selected with high relevance and nonliving with low relevance. Furthermore, the data collected here show that the relevance effect (superiority of high relevance semantic features in concept retrieval) is a strong effect and cannot be explained by any other previously known effects (e.g., typicality, familiarity, etc.).

We mentioned previously that there is no convincing theory that can account for the wealth of empirical data collected on category-specific patients.

To evaluate the potential of our proposal, we compared the predictions of the model with previously published neuropsychological data. A number of critical facts about category-specificity may find an explanation within the framework that we propose.

\section{Impairment for Living}

According to our model, this is a consequence of the intrinsic characteristics of living items that have semantic features that are, on average, less relevant than nonliving ones. If matching for relevance is not carried out carefully, exemplars of living that have lower relevance are likely to be selected, thus reducing response accuracy. This is because random sampling of concepts from categories of living and nonliving results in living having lower relevance and consequently lower accuracy.

\section{Impairment for Nonliving}

A number of patients have been reported to be more impaired on nonliving (18, whereas 61 are reported with specific impairment for living; Capitani et al., 2003). Our explanation is based on the fact that, although the average relevance of living is significantly lower than that of nonliving, there is an overlap between the two distributions. In fact, $16.8 \%$ of nonliving items have an average relevance lower than the mean relevance of living ones. Selecting nonliving items that have lower relevance than those of living ones used as benchmarks will yield greater impairment for nonliving. A corollary of this prediction is that this impairment is less frequent than that for living and this was, in fact, observed.

\section{Opposite Dissociations on the Same Set of Stimuli}

Opposite dissociations on the same set of stimuli. Very few such observations have been reported (Silveri et al.,
1997; Hillis \& Caramazza, 1991; Sartori \& Job, 1988). If we consider that the relevance of semantic attributes is highly influenced by individual differences, we should expect different subjects to behave differently in responding to the same set of stimuli, depending on their particular experience. Individual experience may change the level of knowledge about concepts and, consequently, the constituent relevance values (Tanaka \& Taylor, 1991). We acknowledge that, if two patients matched for variables that may influence knowledge, such as age, level of schooling, and so forth, show opposite dissociations on the same set of stimuli, then this would be problematic for the semantic relevance model. At the moment, in absence of any method for estimating individual premorbid level of knowledge, this explanation must be considered only tentative.

\section{Dissociations within Living: Animals and Vegetables}

Living may not be a single category. In fact, patients with opposite patterns of impairment for animals and vegetables have been reported (Caramazza \& Shelton, 1998; Forde, Francis, Riddoch, Rumiati, \& Humphreys, 1997). The distribution of semantic features according to relevance easily predicts this dissociation. Not controlling for relevance may also yield in this case items with different average relevances.

\section{Degrees of Impairment in Sensory and Functional Knowledge for Living}

Perceptual knowledge in living may be affected to various degrees. Patients with category specificity for living may be more impaired on perceptual semantic features than on nonperceptual ones, but observations of equal deficit have also been reported (Martin, Wiggs, Ungerleider, \& Haxby, 1996). These data are easily explained, in our model, if we consider that living items have perceptual and functional semantic features with similar levels of relevance. Selection of concepts with appropriate levels of relevance may therefore yield all the various patterns of results.

\section{Impaired Performance on Categories with Highly Similar Exemplars (e.g., Musical Instruments)}

It has been observed that living items may be vulnerable because they have exemplars that may be more easily confused with one another. In our model, the accuracy in concept retrieval is reduced, at similar levels of relevance, by semantic similarity between the target and other concepts. Musical instruments, which are characterized by high semantic similarity between exemplars, are expected to have a high rate of misidentification, and this explains why musical instruments may be 
impaired as living in subjects whose object knowledge is spared (Dixon et al., 2001).

Limitations that we can identify in this formulation of our proposal are the following:

1. Individual differences. There is evidence for individual differences among normals. For example Funnell and De Mornay Davies (1996) have identified individual variations in knowledge for animate, plants, and artifacts, and therefore different premorbid levels of knowledge could be at the base of some categoryspecific deficit. Our model, as presented here, does not take into account individual relevance values. In fact, the map of relevance weights of semantic features was computed using descriptions collected from five subjects per concept. While this approach seems sufficient, as a first step, to highlight major problems in normal and abnormal semantic information retrieval, it could be possible to derive for each subject a distinct matrix.

2. Functional neuroanatomy. The model, as presented here, does not address the issue of brain instantiation of semantic processing. Its purely propositional nature, however, could be used as an interpretative framework for those studies that relate semantic processing to gross anatomical regions. At variance with lesion studies, functional imaging studies on normal subjects support the view that different brain regions mediate the identification of living and nonliving things. The data highlight the importance of the inferior temporal lobe for naming animals and the left inferior frontal and middle temporal lobes for naming tools (Martin et al., 1995, 1996; Perani et al., 1995). Anatomical segregation of categories is usually considered as supporting evidence for the domain specific theory. However, if category covaries with relevance, as we have shown, perhaps, it is the latter factor that has neuroanatomical specialization rather than category per se.

While acknowledging that our proposal has some limitations, the findings are convergent and encouraging. Our predictions are based on the distributional statistics deriving from the semantic features map. In summary, concepts differ, in that living have more properties overall and less relevance than do nonliving. Nonliving categories are typically characterized by fewer features with higher relevance. Because low relevance hampers concept retrieval in a "namingto-description" task, this will cause living items to be less accurate, unless relevance is controlled for. We have shown that, while the predicted differences over domains were generally true, there were also considerable variations across categories within domains, which may explain less frequent dissociations, such as selective impairment for nonliving. In this view, it may not be necessary to assume separate domain- or property-based organization within semantic memory to account for even the most selective of semantic deficits.

\section{METHODS}

A general model for the semantic relevance of concepts is outlined, followed by a description of the procedure that we have used to empirically map semantic features to concepts.

\section{Model}

Our main goal was to derive a measure of relevance of semantic features that is intended to represent its contribution to the "core" meaning of a concept. The model we propose boils down to a modified version of the vector space model within the informationretrieval approach (Van Rijsbergen, 1979; Robertson \& Sparck Jones, 1977). The "raw" data from which the process of extraction of the semantic features started were "concept descriptions" as produced by subjects. The final result is a vector of semantic features for every concept, with corresponding relevance weights.

A relevance analysis transforms an $I$ (concepts) $\times J$ (semantic features) intensity data matrix $\mathbf{X}$ into an $I \times J$ relevance model matrix $\mathbf{K}$ that represents the semantic relevance model for the domain under investigation. The entry $x_{i j} \in \mathfrak{R}^{+} \cup 0$ of $\mathbf{X}$ denotes a degree of positive association between Feature $j$ and Concept $i$, whereas the entry $k_{i j} \in \mathfrak{R}^{+} \cup 0$ of $\mathbf{K}$ denotes the relevance of feature $j$ for concept $i$.

The main assumption of our model is that

A1. The relevance matrix $\mathbf{K}$ can be decomposed into an $I \times J$ matrix $\mathbf{L}$ and a $J \times J$ diagonal matrix ${ }^{1}$ $\mathbf{G}$ by means of the matrix product

$$
\mathbf{K}=\mathbf{L G}
$$

In Equation 1, $\mathbf{L}$ represents an $I \times J$ matrix of weights with the entry $l_{i j} \in \mathfrak{R}^{+} \cup 0$ of $\mathbf{L}$ denoting the local importance of Feature $j$ for Concept $i$; hence $\mathbf{L}$ is called the local importance matrix. The main diagonal $\operatorname{diag}(\mathbf{G})$ of $\mathbf{G}$ represents a vector of $J$ weights with the entry $g_{j} \in \mathfrak{R}^{+} \cup 0$ of $\operatorname{diag}(\mathbf{G})$ denoting the overall importance of Feature $j$ for all the $I$ concepts; hence, $\mathbf{G}$ is called the global importance matrix.

$\mathbf{L}$ and $\operatorname{diag}(\mathbf{G})$ can be directly estimated from $\mathbf{X}$ by means of two weighting mappings $\langle\boldsymbol{\phi}, \psi\rangle$,

$$
\mathbf{L}=\boldsymbol{\phi}(\mathbf{X}), \quad \operatorname{diag}(\mathbf{G})=\psi(\mathbf{X})
$$

which act as a linking structure between $\mathbf{X}$ and $\mathbf{K}$. Several weighting schemes may be derived from 
information-retrieval models (Dumais, 1991) and adopted, after appropriate modifications, within a relevance analysis approach. To select the appropriate weighting mappings $\langle\boldsymbol{\phi}, \psi\rangle$, we further assume that

\begin{abstract}
A2. Semantic features with higher within-concept intensity are, in general, more pertinent to that concept.
\end{abstract}

A3. Semantic features whose intensities are equally distributed over all concepts are in general less pertinent than features that are positively associated with only few concepts.

Therefore, we define the following functions:

$$
\begin{gathered}
l_{i j}=\boldsymbol{\phi}\left(x_{i j}\right)=\left\{\begin{array}{llr}
1+\log \left(x_{i j}\right) & \text { if } & x_{i j}>0 \\
0 & \text { if } & x_{i j}=0
\end{array}\right. \\
g_{j}=\psi\left(\mathbf{x}_{. j}\right)=1+\sum_{i=1}^{1} \frac{p_{i j} \cdot \log \left(p_{i j}\right)}{\log (I)}
\end{gathered}
$$

$(\forall i=1, \ldots I ; \forall j=1, \ldots, J)$ with $\mathbf{x}_{j}$ and $p_{i j}$ denoting the $j$ th column of $\mathbf{X}$ and the proportion

$$
p_{i j}=\frac{x_{i j}}{\sum_{i=1}^{I} x_{i j}}
$$

respectively.

Stated more plainly, the function $\boldsymbol{\phi}\left(x_{i j}\right)$ takes the logarithm ${ }^{2}$ of the raw feature intensity $x_{i j}$, thus dampening effects of large differences in intensities (Dumais, 1991). However, notice that $\boldsymbol{\phi}\left(x_{i j}\right)$, being a monotonic function of $x_{i j}$, still preserves the ranking implied by the intensity matrix $\mathbf{X}$.

The mapping $\psi\left(\mathbf{x}_{. \mathbf{j}}\right)$ is based on the information theoretic idea of entropy (or average uncertainty). In particular, the normalized entropy of a feature $j$ $\left(\forall_{j}=1, \ldots, J\right)$ is given by

$$
E\left(\mathbf{x}_{\cdot j}\right)=\sum_{i=1}^{I} \frac{p_{i j} \cdot \log \left(p_{i j}\right)}{\log (I)}
$$

Notice that, adding $E\left(\mathbf{x}_{j}\right) \in[-1,0]$ to 1 (hence, we get $\left.\psi\left(\mathbf{x}_{j}\right)\right)$ assigns minimum weight to features whose intensities are equally distributed over concepts (i.e., where $\left.x_{i j} / \sum_{i=1}^{I} x_{i j}=1 / I\right)$, and maximum weight to features that are positively associated with only few concepts.

Finally, applying Equation 1 , the entry $k_{i j}(\forall i=1, \ldots, I$; $\forall j=1, \ldots, J)$ of the relevance matrix $\mathbf{K}$ takes the form ${ }^{3}$

$$
k_{i j}=l_{i j} g_{i j}
$$

Therefore, a feature that captures the core meaning of a concept will have both high local importance and high global importance.

\section{Mapping Semantic Features to Concepts: An Empirical Procedure}

The procedure that we have followed to extract semantic features and apply the previously described model is the following:

Database of concepts. Cued verbal descriptions of 254 concepts were collected; 43 animals, 15 musical instruments, 53 vegetables, and 143 objects. Concepts belonged to a corpus (Dell'Acqua, Lotto, \& Job, 2000) for which values for major psycholinguistic parameters are available. Our subjects were asked to describe a target concept, and descriptions were cued by the following questions designed to overcome the bias toward certain attributes: (a) give the first free associated word, (b) say how it looks like, (c) say what it is it used for, (d) give any other information you want to give on the concept.

Concept descriptions. Every concept was described by five Italian-speaking subjects. The average length of concept descriptions was 56 words. Concepts where randomly presented to subjects. Using descriptions from different subjects produce an "average" semantic description that eliminates the different levels of semantic richness that subjects may have developed in their experience since different cultural level and direct experience may influence the distribution of semantic features. However, we think that, as a first attempt, this approach is accurate enough to capture broad differences among categories and concepts.

From descriptions to lemmas. Free text, represented by all the descriptions given by subjects to all 254 concepts, underwent word count. Words with a high number of instances in the subjects' definitions (usually function words) and words with a single occurrence were excluded from further analysis. It has been shown (Luhn, 1958) that word frequency furnishes a useful measurement of word significance. If words are ranked according to their frequency in a text, the incapacity to discriminate content reaches a peak at a rank order position of halfway. Lemmatization was then performed by collapsing all variations of basic nouns and verbs under a single attribute (e.g., play, play-s, play-ed, play-ing). Semantic features could be formed of single, two, or three words (e.g., <has a tail $>$, $<$ has a long tail $>$ ).

Computation of the concepts $\times$ semantic features matrix. The words (lemmas) finally remaining may be interpreted as semantic features and were used to produce a concepts $\times$ semantic features matrix in 
Figure 2. Step-by-step procedure for computing relevance values from textual concept descriptions.

\section{Feature Extraction Procedure}

Textual Concept Descriptions

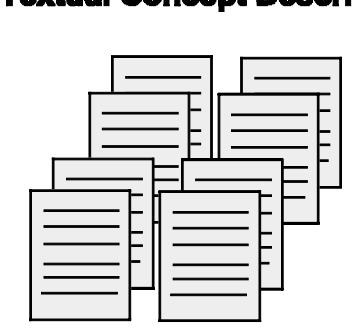

Concept 1 Concept 2 .

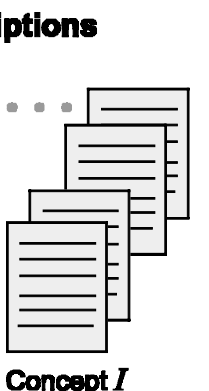

Concopt $I$
Features

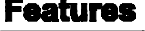

Model Representation

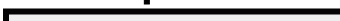

which counts were made for every lemma that appeared in every concept. The original matrix of 254 concepts $\times 8200$ words was reduced to a matrix of 254 concepts $\times 2619$ semantic features. This matrix was then used to derive relevance weights for semantic features.

Computation of relevance weights of semantic features Relevance weights for semantic features were computed using Equation 1 as described above in the Model section.

The steps for deriving relevance of semantic features from concepts are summarized in Figure 2.

\section{Subjects}

\section{Michelangelo and Normal Controls}

This patient has been extensively studied (e.g., Sartori \& Job, 1988) and critically analyzed (e.g., Capitani et al., 2003; Humphreys \& Forde, 2001). In 1984, at the age of 38 years, he was diagnosed with herpes encephalitis. He showed a category-specific impairment for living, which was assessed on several different tasks, including various picture-naming tests, naming-to-verbal-description, drawing from memory, attribute verification, and object decision. The full testing conducted on this patient over the years has recently been summarized (Sartori et al., 2002). His performance was consistent over time and across tasks in showing major impairment of the living category. At the time of the assessment reported here (March 2002), his performance on basic categoryspecificity tests were the following: (a) picture naming: living $=16 / 32$, nonliving $=27 / 32$; (b) reality decision (yes/no): living $=20 / 32$, nonliving $=14 / 16$. The category specific disorder for living was similar in magnitude to that observed in 1988 on the same tests.

Michelangelo's performance was compared with that of six male control subjects of roughly the same age (range 50-70) and cultural level (range 8-12 years of formal education).

\section{Dementia of Alzheimer Type and Normal Controls}

Diagnosis of probable DAT was made according to NINCDS and ADRDA criteria (McKhann et al., 1987). One hundred eight DAT patients were given basic neuropsychological tests and four category specificity tests. Based on the latter (property verification, picture-naming, and two naming-to-description tests), six patients were selected who showed significant and consistent impairment for living over the four tests. We report the data of these six patients in Tables 5 and 6 , together with those of a group of 16 normal controls matched with patients for age and level of education.

The difference between accuracy on nonliving and living was significantly higher in patients with respect to

Table 5. Basic Neuropsychological Information of the DAT Group (with Category Specificity for Living) and of Normal Controls

\begin{tabular}{lccccc}
\hline DAT $(n=6)$ & Age & Education & MMSE & PM & PIM \\
\hline Mean & 77.14 & 4.63 & 18.12 & 1.83 & 1.25 \\
$\begin{array}{l}\text { Standard } \\
\quad \text { deviation }\end{array}$ & 5.43 & 2.25 & 3.62 & 0.25 & 0.84
\end{tabular}

Control $(n=16)$

\begin{tabular}{lccccc} 
Mean & 76.00 & 4.75 & 27.6 & 9.59 & 4.75 \\
$\begin{array}{l}\text { Standard } \\
\text { deviation }\end{array}$ & 4.94 & 1.18 & 1.66 & 2.63 & 1.83 \\
& & & & & \\
$\begin{array}{c}\text { DAT vs. } \\
\text { control }(p)\end{array}$ & .77, ns & $.45, n s$ & $<.0001$ & $<.0001$ & $<.0001$ \\
\hline
\end{tabular}

Age and education are given in years. For both groups: MMSE $(\max =30)$, prose memory $(\mathrm{PM}, \max =28)$, phonemic incidental memory (PIM, $\max =20$ ). 
Table 6. Background Semantic Memory Test Results for the DAT group (with Category Specificity for Living) and of Normal Controls

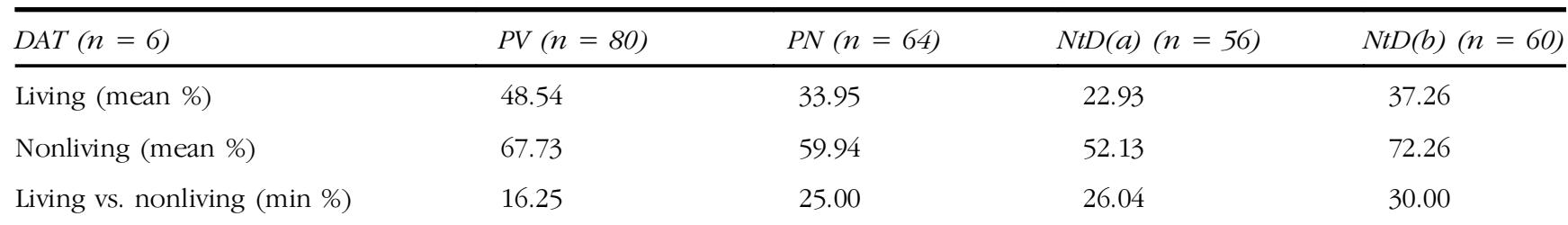

Individual Results

$P V$

PN

$N t D(a)$

$N t D(b)$

MR

Living

Nonliving

$\chi^{2}(1)$

FR

Living

Nonliving

$\chi^{2}(1)$

MS

Living

Nonliving

$\chi^{2}(1)$

$\mathrm{AE}$

Living

Nonliving

$\chi^{2}(1)$

RN

\begin{abstract}
Living
\end{abstract}
Nonliving

$\chi^{2}(1)$

VP

$\begin{array}{lllll}\text { Living } & 21.3 & 46.9 & 6.25 & 23.3 \\ \text { Nonliving } & 37.5 & 71.9 & 33.3 & 56.7 \\ \chi^{2}(1) & 4.62, p<.05 & 4.14, p<.05 & 6.79, p<.01 & 6.92, p<.01\end{array}$

\section{Control $(n=16)$}

Living (mean \%)

Nonliving (mean \%)

Living vs. nonliving (range \%)

DAT vs. control
21.9

46.9

4.42, $p<.05$

40.6

65.6

4.02, $p<.05$

15.6

46.9

7.28, $p<.01$

47.5

65.6

4.66, $p<.05$

52.5

68.8

3.92, $p<.05$

4.01, $p<.05$

3.97, $p<.05$

6.67, $p<.01$
54.2

28.1
43.3

73.3

5.54, $p<.05$

30

63.3

6.68, $p<.01$

33.3

80

6.65, $p<.01$

36.7

73.3

4.36, $p<.05$

56.7

86.7

6.64, $p<.05$

$\mathrm{PV}=$ property verification, $\mathrm{PN}=$ picture naming, $\mathrm{NtD}(\mathrm{a})=$ naming-to-definition (Lambon Ralph, Howard, Nightingale, \& Ellis, 1998, adapted and translated), $\mathrm{NtD}(\mathrm{b})=$ naming-to-definition (Bisiacchi \& Mondini, personal communication). 
normal subjects. This pattern was observed in all four background semantic tests.

The six DAT patients therefore showed a clear, well-defined, category-specific deficit that could be observed in a variety of semantic tasks, such as property verification, picture-naming, and naming-to-description. This category effect was consistently shown by all patient: The minimum magnitude of the impairment for living observed in them was well outside the range of the corresponding figure observed in normal subjects.

Reprint requests should be sent to Giuseppe Sartori, Dipartimento di Psicologia Generale, Universitá di Padova, via Venezia 8, 35100 Padova, Italy, or via e-mail: giuseppe.sartori@unipd.it.

\section{Notes}

1. A matrix $\mathbf{A}$ is a diagonal matrix if (a) $\mathbf{A}$ is a square matrix and (b) $a_{i j}=0$ whenever $i \neq j$.

2. In this article, all logs are base 2 .

3. As $\mathbf{G}$ is a diagonal matrix, Equation 7 can be considered in place of the standard product $k_{i j}=\sum_{b=1}^{J} l_{i b} g_{h j}$.

\section{REFERENCES}

Capitani, E., Laiacona, M., Mahon, B., \& Caramazza, A. (2003). What are the facts of semantic category-specific deficits? A critical review of the clinical evidence. Cognitive Neuropsycholog, 20, 213-261.

Caramazza, A., \& Shelton, J. R. (1998). Domain-specific knowledge systems in the brain: The animate-inanimate distinction. Journal of Cognitive Neuroscience, 10, 1-34.

Dell'Acqua, R., Lotto, L., \& Job, R. (2000). Naming time and standardized norms for the Italian PD/DPSS set of 266 pictures: Direct comparisons with American, English, French, and Spanish published databases. Behaviour Research Methods, Instrumentation and Computers, 32, 588-612.

De Renzi, E., \& Lucchelli, F. (1994). Are semantic systems separately represented in the brain? The case of living category impairment. Cortex, 30, 3-25.

Devlin, J. T., Gonnerman, L. M., Andersen, E. S., \& Seidenberg, M. S. (1998). Category-specific semantic deficits in focal and widespread brain damage: A computational account. Journal of Cognitive Neuroscience, 10, 77-94.

Dixon, M. J., Piskopos, K., \& Schweizer, T. A. (2001). Musical instrument naming impairments: The crucial exception to the living/non-living dichotomy in category specific agnosia. Brain and Cognition, 43, 158-164.

Dumais, S. T. (1991). Improving the retrieval of information from external sources. Behaviour Research Methods, Instrumentation and Computers 23, 229-236.

Durrant-Peatfield, M. R., Tyler, L. K., Moss, H. E., \& Levy, J. P. (1997). The distinctiveness of form and function in category structure: A connectionist model. In M. G. Shafto \& P. Langley (Eds.), Proceedings of the Nineteenth Annual Conference of the Cognitive Science Society (pp. 193-198). Mahwah, NJ: Erlbaum.

Forde, E. M. E., Francis, D., Riddoch, M. J., Rumiati, R., \&
Humphreys, G. W. (1997). On the links between visual knowledge and naming: A single case study of a patient with a category-specific impairment for living things. Cognitive Neuropsychology, 14, 403-458.

Funnell, E., \& De Mornay Davies, P. (1996). JBR: A reassessment of concept familiarity and a category-specific disorder for living things. Neurocase, 2, 461-474.

Funnell, E., \& Sheridan, J. (1992). Categories of knowledge? Unfamiliar aspects of living and nonliving things. Cognitive Neuropsychology, 9, 135-153.

Garrard, P., Lambon Ralph, M. A. Hodges, J. R., \& Patterson, K. E. (2001). Prototypicality, distinctiveness and intercorrelation: Analyses of the semantic attributes of living and nonliving concepts. Cognitive Neuropsychology, 18, $125-174$.

Hillis, A. E., \& Caramazza, A. (1991). Category specific naming and comprehension impairment: A double dissociation. Brain, 114, 2081-2094.

Kintsch, W. (2001). Predication. Cognitive Science, 25, 173-202.

Hodges, J. R., Graham, N., \& Patterson, K. (1995). Charting the progression in semantic dementia: Implications for the organisation of semantic memory. Memory, 3, 463-495.

Humphreys, G. W., \& Forde, E. M. E. (2001). Hierarchies, similarity, and interactivity in object recognition: "Category-specific" neuropsychological deficits. Behavioural and Brain Sciences, 24, 453-509.

Lambon Ralph, M. A., Howard, D., Nightingale, G., \& Ellis, A. (1998). Are living and non-living category specific deficits causally linked to impaired perceptual and associative knowledge? Evidence from a category-specific double dissociation. Neurocase, 4, 311-338.

Luhn, H. P. (1958). The automatic creation of literature abstracts. IBM Journal Research and Development, 2, $159-165$.

Martin, A., Haxby, J. V., Lalonde, F. M., Wiggs, C. L., Ungerleider, L. G. (1995). Discrete cortical regions associated with knowledge of color and knowledge of action. Science, 270, 102-105.

Martin, A., Wiggs, C. L., Ungerleider, L. G., \& Haxby, J. V. (1996). Neural correlates of category-specific knowledge. Nature, 379, 649-652.

McKhann, G., Drachman, D., Folstein, M., Katzman, R., Price, D., \& Stadan, E. M. (1987). Clinical diagnosis of Alzheimer disease: Report of the NINCDS-ADRDA work group. Neurology, 34, 939-944.

Perani, D., Schnurt, T., Tettamanti, M., Gorno-Tempini, M. L., Cappa, S. E., \& Fazio, F. (1995). Different neural systems for the recognition of animals and manmade tools. Neuro Report, 6, 1637-1641.

Robertson, S. E., \& Sparck Jones, K. (1977). Relevance weighting of search terms. Journal of the American Society of Information Sciences, 27, 129-146.

Sacchett, C., \& Humphreys, G. W. (1992). Calling a squirrel a squirrel but a canoe a wigwam: A category specific deficit for artefactual objects and body parts. Cognitive Neuropsychology, 9, 73-86.

Sartori, G., \& Job, R. (1988). The oyster with four legs: A neuropsychological study on the interaction of visual and semantic information. Cognitive Neuropsychology, 5, 105-132.

Sartori, G., Job, R., \& Zago, S. (2002). A case of domain specific semantic deficit. In E. Forde \& G. Humphreys, (Eds.), Category specificity in brain and mind (pp.25-49). Hove: Psychology Press.

Silveri, M. C., Gainotti, G., Perani, D. Cappelletti, J. Y., 
Carbone, G., \& Fazio, F. (1997). Naming deficit for non-living items: Neuropsychological and PET study.

Neuropsychologia, 35, 359-367.

Small, S., Hart, J. Nguyen, T., \& Gordon, H. (1995). Distributed representation of semantic knowledge in the brain. Brain, 118, 441-453.

Stewart, F., Parkin, A. J., \& Hunkin, N. M. (1992). Naming impairment following recovery from herpes simplex encephalitis: Category specificity? Quarterly Journal of Experimental Psychology, 44a, 261-284.

Tanaka, J. W., \& Taylor, M. (1991). Object categories and expertise: Is the basic level in the eye of the beholder? Cognitive Psychology, 23, 457-482.
Tversky, A. (1977). Features of similarity. Psychological Review, 84, 327-352.

Tyler, L. K., Moss, H. E., Durrant-Peatfield, M., \& Levy, J. (2000). Conceptual structure and the structure of the concepts: A distributed account of category specific deficits. Brain Language, 75, 195-231.

Tyler, L., \& Moss, H. (1997). Functional properties of concepts: Studies of normal and brain damaged patients. Cognitive Neuropsychology, 14, 511-545.

Van Rijsbergen, C. J. (1979). Information Retrieval. London: Butterworths.

Warrington, E., \& Shallice, T. (1984). Category-specific semantic impairments. Brain, 107, 829-854. 